\title{
A Case of Idiopathic Thrombocytopenic Purpura during Pregnancy
}

\author{
Maryam Moradi ${ }^{1}$, Fatemeh Chorli ${ }^{2}$, ${ }^{*}$ Leila Asadi $^{1}$ \\ ${ }^{1}$ Reproductive Health and Midwifery Counseling Research Center, Golestan University of Medical \\ Sciences, Gorgan, Iran ${ }^{2}$ Department of Nursing, Golestan University of Medical Sciences, Gorgan, Iran
}

\begin{abstract}
Background: Idiopathic thrombocytopenic purpura (ITP) is one of the common causes of thrombocytopenia in the first and second trimesters of pregnancy. Herein, we report a pregnant woman with ITP with the aim to review the latest evaluation and treatment methods for ITP in the third trimester of pregnancy. Case description: The patient was a 23year-old G2 P1 woman with gestational age of 33 weeks and 4 days, who was referred to hospital due to reduced platelet count $(21,000)$ in the pregnancy screening tests. The patient was subjected to splenectomy after 3 weeks at gestational age of 36 weeks and 4 days, and cesarean section was performed concurrently to terminate the pregnancy. The patient was transferred to the ICU after surgery and was stable. The wound site was normal. Within a few days after the surgery, platelet levels increased to 80,000. Finally, the patient was discharged in a good general condition two weeks after the cesarean section, and was prescribed to take $5 \mathrm{mg}$ prednisolone daily. Conclusion: ITP should be considered in all patients who are first diagnosed with single thrombocytopenia during pregnancy, especially if the condition is detected before the third trimester and with platelet count of less than 50,000. Depending on the course of the disease, patients should be thoroughly monitored for platelet levels for timely treatment.
\end{abstract}

KEYWORDS: Pregnancy, Idiopathic thrombocytopenic purpura, Platelet

*Correspondence: Leila Asadi, Address: Reproductive Health and Midwifery Counseling Research Center, Golestan Universiy of Medical Sciences, Gorgan-Sari Highway, Gorgan, Iran, Telephone: +98-9356518775, Email: asadi.midwife@gmail.com

\section{INTRODUCTION}

Thrombocytopenia is the most prevalent hematological disorder during pregnancy that affects about $10 \%$ of all pregnancies. Idiopathic thrombocytopenic purpura (ITP) is one of the common causes of thrombocytopenia in the first and second trimester of pregnancy (1). This condition is also known as immune thrombocytopenic purpura. According to international epidemiological studies on adults, the estimated incidence rate of this condition is 1.6-2.7 cases per 100,000 individuals per year (2).

Contrary to gestational thrombocytopenia that accounts for about $80 \%$ of all thrombocytopenia incidents in pregnancy, the incidence of ITP is 2 cases per 1000 pregnancies and accounts for about $5 \%$ of all pregnancy-related thrombocytopenia incidents (3). ITP is not related to other causes of thrombocytopenia and is an autoimmune disorder. Its pathogenesis is associated with anti-platelet antibodies, particularly a set of $\operatorname{IgG}$ autoantibodies against one or more platelet glycoproteins. These auto-IgGs are responsible for the early destruction of platelets by macrophages in the spleen and other components of the reticuloendothelial system. Consequently, antibody-coated platelets are prematurely degraded by the reticuloendothelial system, especially in the spleen (1). Since specific clinical and sensitive laboratory tests are not able to detect membrane autoantibodies, ITP is usually diagnosed as single thrombocytopenia with normal or high levels of megakaryocytes and absence of splenomegaly (4). Most women with ITP have mild to moderate thrombocytopenia and approximately $30-35 \%$ of the cases require intervention during pregnancy (3). The disorder can be associated with both fetal and maternal morbidity and mortality (5).

In a study in England, the prevalence of thrombocytopenia was found to be $3.6 \%$, but the mothers in this study had no sign of bleeding (6). In a study in China, $11.7 \%$ of patients had thrombocytopenia and severe bleeding during labor, and $13.2 \%$ of newborns were thrombocytopenic (7). Two studies in Iran reported the prevalence of thrombocytopenia as 8.8\% (8) and 5.2\% (9). Pregnant women with thrombocytopenia are at risk of recurrence and the early diagnosis 
of the disease helps reduce its complications. We herein report a pregnant woman with ITP with the aim to review the latest evaluation and treatment methods for ITP in the third trimester of pregnancy.

\section{CASE PRESENTATION}

The patient was a 23-year-old G2 P1 woman with gestational age of 33 weeks and 4 days, who was referred to hospital due to reduced platelet count $(21,000)$ in the pregnancy screening tests. She mentioned history of lowered platelet count since the beginning of pregnancy and has been treated for suspected lupus in the past two months. There was a long history of ecchymosis, petechiae, purpura and bleeding gum. Systolic and diastolic blood pressure was $120 \mathrm{mmHg}$ and $60 \mathrm{mmHg}$, respectively. Physical examination was normal and there was no sign of vaginal bleeding or uterine contraction, and heart auscultation of fetus was normal. The patient was hospitalized with diagnosis of ITP. The patients received $60 \mathrm{mg}$ prednisolone daily and $200 \mathrm{mg}$ hydroxychloroquine twice a day. A gynecologist and a hematologist visited the patient and requested daily complete blood count with differential. Two packed cells were reserved for the patient. On the following day, the patient's platelet level reached 25,000 and his hemoglobin reached $8.5 \mathrm{~g} / \mathrm{dL}$ (Table 1). The patient was put on total bed rest and was prohibited from intramuscular injections. A 24-hour urine sample was collected and there was no proteinuria. Abdominal ultrasound was performed and the spleen and liver had normal size. The results of fetal health assessment were normal. Perinatology consultation was done and there was no indication of pregnancy termination. The physician ordered monitoring for signs of preeclampsia and hypertension. In the case of emergence of the symptoms and failure to respond to hematologic treatments with pulsed corticosteroid and intravenous immunoglobulin (IVIG) and further drop of platelet count, the pregnancy termination would be considered. Later, platelet levels reached 19,000 and the patient was treated with corticosteroids ( $1 \mathrm{~g}$ intravenous methylprednisolone vial) daily for three days. In consecutive measurements, the patient's platelet count was within the 30,000 range. IVIG was started for the patient and continued for two days. Lupus and antiphospholipid syndrome were negative in the rheumatologic tests. Considering the lack of increase in the patient's platelet count $(32,000)$ after drug therapies and the risk of drug resistance as well as increased possibility of spontaneous bleeding with a platelet count of less than 50,000, the patient was subjected to splenectomy three weeks later at gestational age of 36 weeks and 4 days. Cesarean section was done simultaneously for termination of the pregnancy. The patient was transferred to the ICU after surgery while stable and the wound site was normal. Within a few days after the surgery, platelet levels increased to 80,000. Finally, the patient was discharged in a good general condition two weeks after the cesarean section, and was prescribed $5 \mathrm{mg}$ prednisolone daily. 
Table 1. Platelet level changes over the hospitalization period

\begin{tabular}{|c|c|}
\hline Data & Platelet count \\
\hline $06 / 09 / 2017$ & 30000 \\
\hline $07 / 09 / 2017$ & 25000 \\
\hline $10 / 09 / 2017$ & 36000 \\
\hline $12 / 09 / 2017$ & 30000 \\
\hline $15 / 09 / 2017$ & 36000 \\
\hline $17 / 09 / 2017$ & 34000 \\
\hline $18 / 09 / 2017$ & 30000 \\
\hline $26 / 09 / 2017$ & 30000 \\
\hline $27 / 09 / 2017$ & 70000 \\
\hline $02 / 10 / 2017$ & 80000 \\
\hline
\end{tabular}

\section{DISCUSSION}

Compared to gestational thrombocytopenia, ITP can occur in all trimesters of pregnancy and is characterized by low platelet count with hemorrhagic status. Initial detection of thrombocytopenia in the first trimester or early second trimester makes the diagnosis of ITP more likely (3). Our case mentioned the history of prior low platelet counts since early pregnancy. The clinical symptoms of ITP are shared between pregnant and nonpregnant individuals. Patients with ITP may be sometimes diagnosed with severe thrombocytopenia accompanied with bruising, bleeding and petechiae (10). ITP is usually accompanied with petechiae, ecchymosis, mucosal hemorrhage (gum, nose, urinary tract and digestive tract) and menorrhagia associated with platelet counts (1). Our case had a long history of ecchymosis, petechiae, purpura and bleeding gum. To diagnose other factors associated

with thrombocytopenia, preeclampsia, systemic lupus erythematosus and antiphospholipid syndrome should be ruled out (10). In this patient, additional tests and examinations ruled out preeclampsia and rheumatologic disorders. Similar to treatment in non-pregnant women, corticosteroids and IVIG are acceptable for the treatment of ITP during pregnancy. Generally, corticosteroids are the first line therapy for pregnant women
With ITP, and IVIG is applied to rapidly increase platelet count. Splenectomy may be considered as the second-line therapy for pregnant women with refractory ITP (1). In our case, despite the drug therapy with corticosteroids and IVIG, platelet levels did not increase. Thus, due to the lack of positive effects of the above therapeutic methods, the patient was ultimately subjected to splenectomy and cesarean section.

\section{CONCLUSION}

ITP should be considered in all patients who are first diagnosed with single thrombocytopenia during pregnancy, especially if the condition is detected before the third trimester and with platelet count of less than 50,000. Depending on the course of the disease, patients should be thoroughly monitored for platelet levels for timely treatment.

\section{REFERENCES}

1. Myers B. Diagnosis and management of maternal thrombocytopenia in pregnancy. $\mathrm{Br} \mathrm{J}$ Haematol. 2012;158:3-15.

2. Abrahamson PE, Hall SA, Feudjo-Tepie M, et al. The incidence of idiopathic thrombocytopenic purpura among adults: a population-based study 
and literature review. Eur J Haematol. 2009;83:83-89.

3. Gernsheimer T, James A H, Stasi R. How I treat thrombocytopenia in pregnancy. Blood. 2013;121:38-47.

4. Thienelt CD, Calverley DC. Thrombocytopenia Caused by Immunologic Platelet Distruction. Wintrobes Clinical Hematology 2009;II(Chapter 51).

5. Delmas Y, Helou S, Chabanier P, Ryman A, Pelluard F, Carles D, et al. Incidence of obstetrical thrombotic thrombocytopenic purpura in a retrospective study within thrombocytopenic pregnant women. A difficult diagnosis and a treatable disease. BMC Pregnancy Childbirth 2015; 15:137.

6. Matthews JH, Benjamin S, Gill DS, Smith NA. Pregnancy-associated thrombocytopenia: definition, incidence and natural history. Acta Haematol 1990; 84(1): 24-29.

7. Zhang MM, Jiang B. Clinical analysis of 60 cases of pregnancy with thrombocytopenia. Zhonghua Fu Chan Ke Za Zhi 1992; 27(4): 224226, 250-251.

8. Modarresnejad V, Motamedi B. Study of the Prevalence of Thrombocytopenia and Factors Affecting it in Kerman. Asrar 2001; 9(2): 4- 10 (Persian).

9. Yazdani Sh, Bouzari Z, Sedaghat S., Abedi Samaquosh, Farajnejad K. Prevalence of thrombocytopenia in pregnancy and its related factors. IJOGI, Vol. 19, No. 32, pp. 20-24, Nov 2016.

10. Stavrou E, McCrae KR. Immune thrombocytopenia in pregnancy. Hematol Oncol Clin North Am 2009;23:1299-316. 